\title{
Conceptual question response times in Peer Instruction classrooms
}

\author{
Kelly Miller, ${ }^{1, *}$ Nathaniel Lasry, ${ }^{2}$ Brian Lukoff, ${ }^{1}$ Julie Schell, ${ }^{1}$ and Eric Mazur ${ }^{1}$ \\ ${ }^{1}$ Department of Physics and Division of Engineering and Applied Sciences, \\ Harvard University, Cambridge, Massachusetts 02138, USA \\ ${ }^{2}$ Department of Physics, John Abbott College, Montreal, Quebec H9X 3L9, Canada
}

(Received 31 March 2014; published 19 August 2014)

\begin{abstract}
Classroom response systems are widely used in interactive teaching environments as a way to engage students by asking them questions. Previous research on the time taken by students to respond to conceptual questions has yielded insights on how students think and change conceptions. We measure the amount of time students take to respond to in-class, conceptual questions [ConcepTests (CTs)] in two introductory physics courses taught using Peer Instruction and use item response theory to determine the difficulty of the CTs. We examine response time differences between correct and incorrect answers both before and after the peer discussion for CTs of varying difficulty. We also determine the relationship between response time and student performance on a standardized test of incoming physics knowledge, precourse self-efficacy, and gender. Our data reveal three results of interest. First, response time for correct answers is significantly faster than for incorrect answers, both before and after peer discussion, especially for easy CTs. Second, students with greater incoming physics knowledge and higher self-efficacy respond faster in both rounds. Third, there is no gender difference in response rate after controlling for incoming physics knowledge scores, although males register significantly more attempts before committing to a final answer than do female students. These results provide insight into effective CT pacing during Peer Instruction. In particular, in order to maintain a pace that keeps everyone engaged, students should not be given too much time to respond. When around $80 \%$ of the answers are in, the ratio of correct to incorrect responses rapidly approaches levels indicating random guessing and instructors should close the poll.
\end{abstract}

DOI: 10.1103/PhysRevSTPER.10.020113

PACS numbers: 01.40.Fk

\section{INTRODUCTION}

When used effectively, classroom response systems (CRSs) can facilitate student engagement in science classrooms in ways that would not be possible otherwise [1,2]. From low-technology versions, such as flash cards or whiteboards, to higher-technology, Web-based response systems, such as Poll Everywhere or Learning Catalytics, the popularity of CRSs has also opened up an active area for educational researchers to pursue questions about their use and student learning outcomes. Peer Instruction is one popular research-based instructional strategy that leverages the power of CRSs to promote student learning [3]. In Peer Instruction, students use CRSs to respond to ConcepTests (CTs) both before and after discussing their answers with their peers for 2-5 minutes [4]. ConcepTests are short conceptual questions that focus on a single topic [3]. During Peer Instruction, students first respond individually to a CT and then respond a second time to the same CT after discussing their responses with a peer. Instructors

\footnotetext{
*kmiller@seas.harvard.edu

Published by the American Physical Society under the terms of the Creative Commons Attribution 3.0 License. Further distribution of this work must maintain attribution to the author $(s)$ and the published article's title, journal citation, and DOI.
}

can use CT response data to inform teaching decisions in real time. Researchers suggest that Peer Instruction is a superior teaching strategy in promoting student conceptual understanding and problem solving skills compared to the traditional lecture [3-8]. The purpose of this research is to gain insight into student thinking in Peer Instruction environments using one specific unit of analysis related to CTs: response time.

Despite the fact that Peer Instruction is a research-based pedagogy shown to be highly effective in promoting student learning, there is room for improvement particularly in implementation. Response times can provide insight into student thinking during Peer Instruction. Previous research has shown that when students answer conceptual questions with misconceptionlike responses, they tend to respond more quickly than those answering correctly [9] With this insight, instructors can improve the implementation of Peer Instruction. For example, an examination of response time could inform CT pacing and help determine the optimal amount of time instructors should keep questions open for student response. Providing instructors with guidelines for appropriate CT pacing allows them to implement Peer Instruction more efficiently by optimizing class time.

We pose two research questions. First, what are the response time differences between correct and incorrect 
answers, before and after peer discussion for CTs of varying difficulty? Second, what is the relationship between response time and student performance on a standardized test of incoming physics knowledge, precourse self-efficacy, and gender?

Physics education researchers have previously conducted studies of response times to conceptual questions to gain insight into student learning. Using standardized conceptual questions delivered as a pre-post course conceptual survey, Lasry et al. showed that response times for incorrect answers are longer than for correct responses [10]. Our study differs from Lasry et al. in that we examine inclass formative conceptual questions versus pre-post course surveys. Richardson and O'Shea examined the relationship between gender and CT response time in a Peer Instruction classroom and found no statistically significant difference in response time between males and females [11]. However, Richardson and O'Shea also found that males were more likely than females to change their answer within a single round before committing to a final response [11]. In this study of CT response times, we extend this work by combining response times with student learning data to gain further insight into student thinking in Peer Instruction environments.

\section{METHODS}

We collected student responses and response times for ConcepTests from one semester in two introductory electricity and magnetism classes at Queens University (QU) (Kingston, Ontario; $N=48$ ) and Harvard University (HU) (Cambridge, Massachusetts; $N=93$ ). Both classes used Peer Instruction with between five and 15 CTs per class. Over the course of the semester, $101 \mathrm{CTs}$ were given at Queens and 74 at Harvard. In both classes, students did not receive credit for getting the answer right. Credit was only given for responding to the questions. Students answered each CT in two rounds of questioning by entering their responses via a classroom response system (iClicker at Queens University and Learning Catalytics at Harvard). The CRS recorded a time stamp for each student's final response. Response times were computed as the difference between the time the question was delivered to the students (via the CRS) and the time of each student's final response. Regardless of the number of responses a student entered for a single question, only the time to the final response was recorded. For each question, we analyzed the time taken to respond before and after the discussion. The response time before the discussion is the amount of time it takes students to respond individually to the question the first time they see it. After students discuss answers with each other, the instructor presents the question again and opens the polling for a second time. The response time after the discussion is the amount of time that students take to respond after the polling is opened the second time. We did not constrain how long each question would stay open because some questions take longer to parse than others. We also chose to give each instructor the control of how much time each question stayed open. Students often continue their discussion past the time when the poll is reopened for the second round. Consequently, some questions stayed open much longer than others. In some rare cases, students were given more than 5 minutes to respond to a single question, especially in the second round. To control for the variability in response times across questions, we focus our analysis on the difference in the time taken to respond correctly and incorrectly. The iClicker system used at Queens also records the number of times a student responds to a CT before registering their final answer and, for this subset of the data, we include this in our analysis.

The Conceptual Survey of Electricity and Magnetism (CSEM) [12] and a self-efficacy survey were both administered twice in each class, once as pretests at the beginning of the semester and again as post-tests at the end of the semester. Self-efficacy is a person's belief that they can be successful when performing a situation-specific task [13], and students' self-efficacy has been shown to be a strong predictor for perseverance and success in science [14]. The self-efficacy survey used in this study was developed at Harvard, and based on the Sources of Self-efficacy in Science Courses (SOSESC) survey [14]. This survey asked students to rank, on a five-point scale, the extent to which they believe they will be successful in a number of physicsrelated tasks (e.g., how successful will they be solving difficult physics problems or communicating physics successfully to a peer).

A two-parameter item response model [15] was applied to the first round of responses across all items to determine the difficulty of each item ( $b$ parameter). Items were grouped into two categories according to difficulty. Items for which $b<0$ were classified as easy and items with $b \geq 0$ were classified as hard. In the Queens data set, 59 out of the $101 \mathrm{CTs}$ were classified as easy and the remaining 42 as hard. In the Harvard data set, 38 of the 74 CTs were classified easy and the remaining 36 as hard.

\section{RESULTS}

Table I shows the average time taken for correct and incorrect answers on ConcepTests both before $\left\langle t_{\text {before }}\right\rangle$ and

TABLE I. Average time taken for correct and incorrect answers on ConcepTests, before and after Peer Instruction. $* * p<0.0001$, $* p<0.005$. [Harvard University (HU), Queen's University (QU).]

\begin{tabular}{lccccc}
\hline \hline & \multicolumn{2}{c}{$\left\langle t_{\text {before }}\right\rangle(\mathrm{s})$} & & \multicolumn{2}{c}{$\left\langle t_{\text {after }}\right\rangle(\mathrm{s})$} \\
\cline { 2 - 3 } \cline { 5 - 6 } & $\mathrm{HU}$ & $\mathrm{QU}$ & & $\mathrm{HU}$ & $\mathrm{QU}$ \\
\hline Correct & 70.9 & 26.9 & & 95.3 & 64.3 \\
Incorrect & 75.8 & 35.5 & & 102.3 & 69.9 \\
Difference & $4.9^{*}$ & $8.6^{* *}$ & & $7.0^{*}$ & $5.6^{*}$ \\
\hline \hline
\end{tabular}



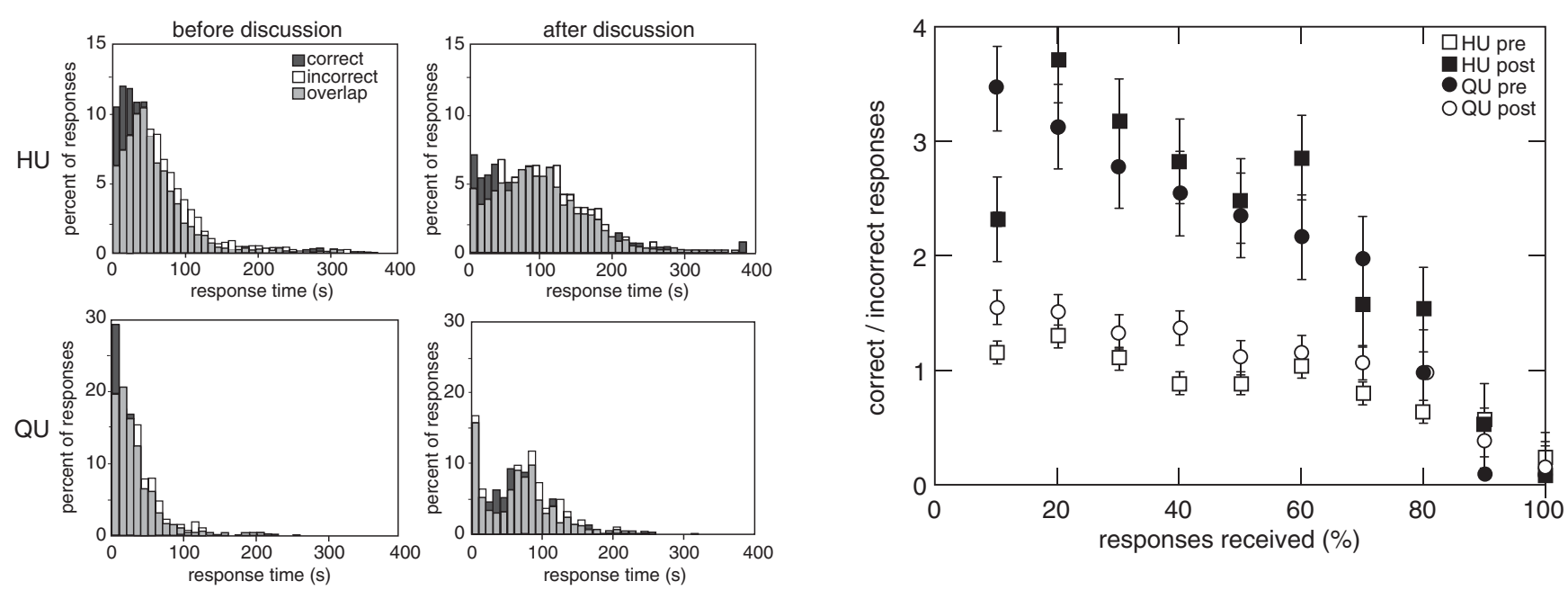

FIG. 1. Distributions of ConcepTest response times before and after peer discussion.

after $\left\langle t_{\text {after }}\right\rangle$ peer discussion at Queen's and Harvard University. Before peer instruction, the average response time is $20 \%-30 \%$ shorter for correct answers than for incorrect answers $(p<0.001)$. After peer discussion, the average response time is about $10 \%$ shorter for correct answers than for incorrect answers $(p<0.01)$.

We also analyzed the average response time difference between incorrect and correct responses $\left\langle t_{\text {incorr }}\right\rangle-\left\langle t_{\text {corr }}\right\rangle$ for each individual question. Of the 175 questions posed in this study 27 had a statistically significant difference (at $p<0.05$ ) where $\left\langle t_{\text {incorr }}\right\rangle>\left\langle t_{\text {corr }}\right\rangle$, three questions had a statistically significant difference (at $p<0.05$ ) where $\left\langle t_{\text {incorr }}\right\rangle\left\langle\left\langle t_{\text {corr }}\right\rangle\right.$, and 145 had no significant difference between $\left\langle t_{\text {incorr }}\right\rangle$ and $\left\langle t_{\text {corr }}\right\rangle$. Therefore, for the majority of the questions with a significant time difference, incorrect responses are not misconceptionlike in nature because students take longer to provide an incorrect answer than the correct one.

Figure 1 shows histograms for responses as a function of ConcepTest response time before and after peer discussion. Correct responses (dark gray) and incorrect responses (white) are expressed as a percentage of all responses over all the ConcepTest questions posed during the semester. The light gray area represents the overlap of the white and dark gray bars. The top two histograms represent data from

FIG. 2. Ratio of correct to incorrect responses as a function of the percentage of responses received.

Harvard and the bottom two histograms represent data from Queen's. Figure 1 illustrates the shorter time scale with which correct responses are given compared to incorrect responses. Given that there was no set amount of time that the polling stayed open, we find some questions were left open for more than 5 minutes.

Figure 2 shows the ratios of correct to incorrect responses plotted as a function of the percent of students in the class who have entered their response. Ratios of correct to incorrect responses are shown for responses before the discussion (pre) and after the discussion (post) for questions from both the Harvard classroom and the Queens classroom. Error bars represent the standard error of the mean across each of the four ratios. Figure 2 shows that as more students respond, the proportion of correct answers decreases compared to the proportion of incorrect answers, both before and after the peer discussion. When about $80 \%$ of the students in the class have responded, the ratio of correct to incorrect answers rapidly approaches levels indicating random guessing by the students.

Table II displays the average response times for correct and incorrect answers for questions determined to be easy and hard. The difference in response time was determined by subtracting the average response time for correct answers from the average response time for incorrect answers. The difference is positive for easy and hard

TABLE II. Average time taken for correct and incorrect answers on ConcepTests classified as easy and hard, before and after peer discussion. $* * p<0.0001, * p<0.005$.

\begin{tabular}{|c|c|c|c|c|c|c|c|c|}
\hline & \multicolumn{4}{|c|}{$\left\langle t_{\text {before }}\right\rangle(\mathrm{s})$} & \multicolumn{4}{|c|}{$\left\langle t_{\mathrm{after}}\right\rangle(\mathrm{s})$} \\
\hline & \multicolumn{2}{|c|}{$\mathrm{HU}$} & \multicolumn{2}{|c|}{$\mathrm{QU}$} & \multicolumn{2}{|c|}{$\mathrm{HU}$} & \multicolumn{2}{|c|}{ QU } \\
\hline & Easy & Hard & Easy & Hard & Easy & Hard & Easy & Hard \\
\hline Correct & 52.9 & 65.0 & 24.9 & 35.3 & 80.5 & 109.9 & 52.2 & 87.9 \\
\hline Incorrect & 62.2 & 69.9 & 29.1 & 36.1 & 101.8 & 113.9 & 60.4 & 89.3 \\
\hline Difference & $9.3 * *$ & 4.9 & $4.2 * *$ & 0.8 & $21.3 * *$ & 4.0 & $8.2 * *$ & 1.4 \\
\hline
\end{tabular}




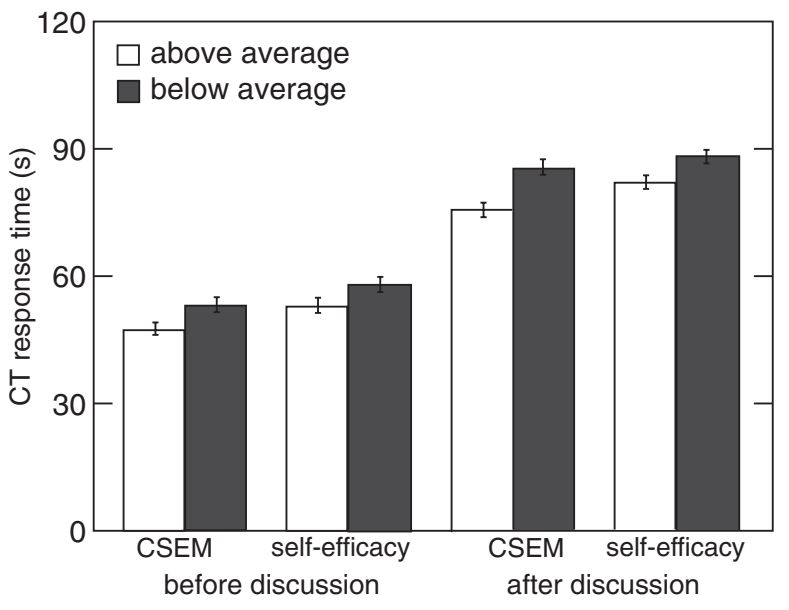

FIG. 3. Average response times before and after discussion for students with below and above average precourse CSEM scores and below and above average precourse self-efficacy scores.
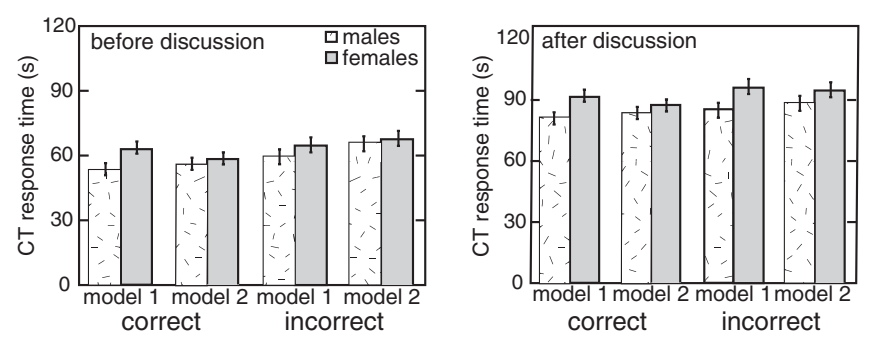

FIG. 4. Difference in response times both before and after discussion. We show both model 1 , which controls only for gender, and model 2, which also controls for precourse CSEM and self-efficacy scores.

questions both before and after the peer discussion indicating that, regardless of the difficulty of the question or whether the response is provided before or after the discussion, students take longer to respond with an incorrect answer than with a correct one. However, this response time difference is only statistically significant for easy questions $(p<0.0001)$. For difficult questions, while the average response time is longer for incorrect answers than for correct answers, the difference is small and not statistically significant. Table II also shows that students take longer to answer hard questions than easy questions, regardless of whether they are answering correctly or incorrectly.

Figure 3 shows that students with more incoming physics knowledge and higher self-efficacy respond faster, both before and after the peer discussion. Figure 3 displays average response times before and after the peer discussion for students in four different groups: (1) below average precourse CSEM scores, (2) above average precourse CSEM scores, (3) below average precourse self-efficacy scores, and (4) above average precourse self-efficacy scores. Students with higher incoming physicsaa knowledge (above average precourse CSEM scores) respond approximately $15 \%(p<0.001)$ faster before the peer discussion, and around 10\% $(p<0.001)$ faster after the peer discussion, than students with lower incoming physics knowledge (below average precourse CSEM scores). Students with above average scores on the precourse self-efficacy survey respond about $10 \%(p<0.001)$ faster both before and after the peer discussion than students with below average scores.

Figure 4 shows male and female student response times for correct and incorrect answers. The first set of bars in each cluster (model 1) displays the response times when controlling for gender only, while the second set of bars (model 2) displays the response times after adding precourse CSEM and self-efficacy scores to model 1 as additional predictor variables. The left-hand plot of Fig. 4 indicates that males respond faster than females before discussion, regardless of whether they have the answer correct or incorrect, but that this difference disappears when students' precourse knowledge and self-efficacy are controlled for. The right-hand plot of Fig. 4 shows that the same is true when considering male and female response times after peer instruction. The regression parameters and significance metrics for both models are displayed in Table III.

Table IV shows that a gender difference exists in the number of attempts students register before committing to a final answer. Compared to female students, male students respond significantly more times before deciding on a final response. The average number of attempts made by males exceeds that made by females both before $\left\langle n_{\text {before }}\right\rangle$ and after

TABLE III. Regression models predicting student response times for correct and incorrect responses both before and after the discussion. $* p<0.05, * * p<0.01, * * * p<0.001$.

\begin{tabular}{|c|c|c|c|c|c|c|c|c|}
\hline & \multicolumn{4}{|c|}{ Before discussion } & \multicolumn{4}{|c|}{ After discussion } \\
\hline & \multicolumn{2}{|c|}{ Correct } & \multicolumn{2}{|c|}{ Incorrect } & \multicolumn{2}{|c|}{ Correct } & \multicolumn{2}{|c|}{ Incorrect } \\
\hline & Model 1 & Model 2 & Model 1 & Model 2 & Model 1 & Model 2 & Model 1 & Model 2 \\
\hline Gender & $9.5^{*}$ & 2.38 & 3.71 & 1.16 & $8.5^{*}$ & 4.9 & $9.9 * *$ & 5.12 \\
\hline Precourse physics knowledge & & $-1.27 * * *$ & & $-0.67 *$ & & $-1.14 * * *$ & & $-1.23 * *$ \\
\hline Precourse self-efficacy & & $0.58 * * *$ & & $0.50 * * *$ & & $0.39 * * *$ & & $0.38 * * *$ \\
\hline $\mathrm{R}^{2}$ & 0.15 & 0.14 & 0.15 & 0.14 & 0.14 & 0.11 & 0.16 & 0.15 \\
\hline RMSE & $62.3 * *$ & 65.4 & 55.5 & 56.7 & 59.7 & 59.6 & 57.4 & 57.9 \\
\hline
\end{tabular}


TABLE IV. Average number of attempts for males and females on ConcepTests, before and after the peer discussion. $* * * p<0.0001$.

\begin{tabular}{lcc}
\hline \hline & $\left\langle n_{\text {before }}\right\rangle$ & $\left\langle n_{\text {after }}\right\rangle$ \\
\hline Males & 2.4 & 3.0 \\
Females & 1.7 & 1.8 \\
Difference & $1.3^{* *}$ & $1.2^{* *}$ \\
\hline \hline
\end{tabular}

$\left\langle n_{\text {after }}\right\rangle$ the peer discussion. Before the peer discussion, male students change their initial response $40 \%$ more times than female students $(p<0.001)$ before deciding on a final response. After the peer discussion, male students change their initial response $65 \%$ more times than female students $(p<0.001)$ before deciding on a final response.

\section{DISCUSSION}

\section{A. Incorrect answers take more time}

Multiple-choice ConcepTests are designed so that the incorrect choices are distractors, that is, incorrect ideas that are commonly held by students. These conceptions are often portrayed as common-sense beliefs that are stable and resistant to instruction [16]. Previous research has shown that when students answer conceptual questions with misconceptionlike responses, they tend to respond more quickly than those answering correctly [9]. In other words, strong distractors should yield quick responses. However, we find that students take longer to respond when they answer incorrectly, suggesting that these choices are not seen as strong distractors that yield automatic responses. Our data therefore suggest that when students select an incorrect answer, it is more likely because they do not know the answer, rather than because they are confident about the wrong answer.

We find that students take longer to respond to more difficult questions. Instructors frequently adjust their pacing based on the fraction of the class that has responded to a question, leaving the poll open longer for more difficult questions. Therefore, the student response rate for a question dictates the length of time the poll for that question is left open, but only up to a point. Instructors often close the poll before $100 \%$ of their class has responded, though, according to our data, rarely when less than $77 \%$ has voted. On average, in the two classrooms used in this study, polls were closed once $91 \%$ of students had responded. Figure 2 shows that once more than $80 \%$ of students have responded, the ratio of correct to incorrect answers is so low that instructors should consider closing the poll. Once $90 \%$ of students have responded, the correct to incorrect ratio is on average $25 \%$, indicating that students are doing no better than random guessing.

We find that the difference in response time between correct and incorrect answers depends on the difficulty of the question. For easy questions, incorrect answers are not automatic; they take significantly longer than correct answers. Students answering easy questions incorrectly are spending time thinking. For harder questions, this does not appear to be the case. When the questions are difficult, the time taken to give an incorrect answer does not differ statistically from the time taken to give a correct answer. Apparently, the correct choice is not as obvious as for easy questions and students take as much time evaluating correct and incorrect options.

Response time for both correct and incorrect answers is significantly longer after peer discussion. Figure 1 shows that after discussing the question with their peers, students take longer to respond to the question than when they respond individually. There are two possible explanations for this finding. It is possible that response times are longer in the second round $\left\langle t_{\mathrm{after}}\right\rangle$ because students take more time to think about the question. Alternatively, it could simply be due to the fact that some students may continue talking after the polling has reopened and that $\left\langle t_{\text {after }}\right\rangle$ includes some of the discussion time.

Our findings are based on CT response time data collected at two different institutions in two different classrooms with instructors who had very different pacing. A comparison of the top two histograms and the bottom two histograms in Fig. 1 illustrates the different pace at which CTs were posed to students at Harvard (top) and to students at Queen's (bottom). On average, the polling in the Harvard classroom was kept open much longer than in the Queen's classroom, both before and after the discussion. Despite this difference in pacing, the same time scale difference emerges between correct and incorrect responses. Regardless of the institution and how long students are given to answer the questions, incorrect answers take more time than correct answers.

\section{B. Response times vary according to students' precourse characteristics}

Figure 3 shows that the students who respond faster are those who know more physics and have a stronger belief in their ability to be successful in a physics course at the beginning of the semester. These students spend less time considering alternative answers; that is, their answers are more automatic. Interestingly, these students answer more quickly, regardless of whether they are answering correctly or incorrectly. This finding suggests that response times are dependent on intrinsic student characteristics as well as cognitive processes.

\section{CTs responses and gender}

In contrast to earlier findings [11], we find that although males respond significantly faster than females, the difference disappears after controlling for self-efficacy and CSEM scores. The gender difference in response times appears to be at least partly attributable to a difference in precourse knowledge and self-efficacy. When answering 
correctly, males respond $20 \%$ faster than females before $(p<0.05)$ the discussion and 10\% faster after $(p<0.05)$ the discussion. When answering incorrectly, the difference is less pronounced. Before the discussion, males answer incorrectly $6 \%$ faster than females (albeit this difference is not significant) and $12 \%$ faster after the discussion $(p<0.05)$. However, when a linear regression model is used to control for precourse knowledge and self-efficacy, gender ceases to be a significant predictor of response times and the gender difference disappears completely.

Our results also show that, compared to female students, male students respond significantly more times before deciding on a final response. This result is consistent with previous findings that males are more likely than females to answer with more attempts [17] and change their answer within a single round before committing to a final response [11].

\section{CONCLUSION}

For two different student populations, we find three interesting results from an analysis of response times to conceptual questions posed in class. The first is that incorrect answers take more time than correct answers, especially for easy questions. This suggests that incorrect responses result from students not knowing the answer, rather than from strongly held misconceptions. The second is that students with greater incoming physics knowledge and higher self-efficacy respond faster, indicating that response times are partly a function of student characteristics. Third, there is no gender difference in response rate when other student characteristics are controlled for. In light of these findings, we recommend that instructors terminate polls once $80 \%$ of the answers are in, because at that point an increasing fraction of students respond by random guessing.

\section{ACKNOWLEDGMENTS}

Several people contributed to the work described in this paper. E. M. and N. L. conceived of the basic idea for this work. K. M., B. L., and E. M. designed and carried out the study, and K. M. analyzed the results. J.S. and B. L. designed and implemented the self-efficacy survey used in this study. E. M. and N. L. supervised the research and the development of the manuscript. K. M. wrote the first draft of the manuscript; all authors subsequently took part in the revision process and approved the final copy of the manuscript. James Fraser and Anneke Timan provided the data from Queens University and were important advisors throughout the study.
[1] D. Bruff, Teaching with Classroom Response Systems: Creating Active Learning Environments (John Wiley \& Sons, New York, 2009).

[2] J. Caldwell, Clickers in the large classroom: Current research and best-practice tips, CBE Life Sci. Educ. 6, 9 (2007).

[3] E. Mazur, Peer Instruction A User's Manual (PrenticeHall, Upper Saddle River, NJ, 1997).

[4] C. H. Crouch and E. Mazur, Peer Instruction: Ten years of experience and results, Am. J. Phys. 69, 970 (2001).

[5] S. B. McKagan, K. K. Perkins, and C. E. Wieman, Reforming a large lecture modern physics course for engineering majors using a PER-based design, AIP Conf. Proc. 883, 34 (2007).

[6] R. N. Cortright, H. L. Collins, S. E. Dicarlo, N. Ronald, and E. Stephen, Peer instruction enhanced meaningful learning: Ability to solve novel problems, Adv. Physiol. Educ. 29, 107 (2005).

[7] A. P. Fagen, Peer Instruction: Results from a Range of Classrooms, Phys. Teach. 40, 206 (2002).

[8] S.P. Rao and S. E. DiCarlo, Peer instruction improves performance on quizzes, Adv. Physiol. Educ. 24, 51 (2000).

[9] A. F. Heckler, T. M. Scaife, and E. C. Sayre, Proceedings of the 32 Annual Conference of the Cognitive Science Society, edited by S. Ohlsson and R. Catrambone
(Cognitive Science Society, Austin, TX, 2010) pp. 139-144.

[10] N. Lasry, J. Watkins, E. Mazur, and A. Ibrahim, Response times to conceptual questions, Am. J. Phys. 81, 703 (2013).

[11] C. T. Richardson and B. W. O'Shea, Assessing gender differences in response system questions for an introductory physics course, Am. J. Phys. 81, 231 (2013).

[12] D. P. Maloney, T. L. O'Kuma, C. J. Hieggelke, and A. Van Heuvelen, Surveying students' conceptual knowledge of electricity and magnetism, Am. J. Phys. 69, S12 (2001).

[13] A. Bandura, Self-efficacy: Toward a unifying theory of behavioral change, Psychol. Rev. 84, 191 (1977).

[14] H. Fencl and K. Scheel, Pedagogical approaches, contextual variables, and the development of student selfefficacy in undergraduate physics courses, AIP Conf. Proc. 720, 173 (2004).

[15] D. Thissen and S. Lynne, A taxonomy of item response models, Psychometrika 51, 567 (1986).

[16] I. Abou Halloun and D. Hestenes, The initial knowledge state of college physics students, Am. J. Phys. 53, 1043 (1985).

[17] G. Kortemeyer, Gender differences in the use of an online homework system in an introductory physics course, Phys. Rev. ST Phys. Educ. Res. 5, 010107 (2009). 